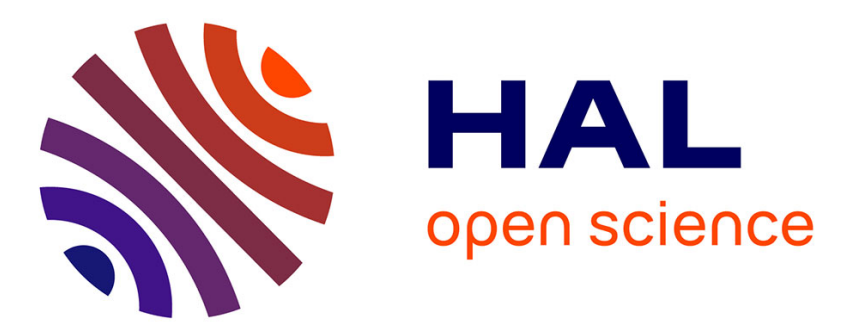

\title{
Quantifying the energetic cost of food quality constraints on resting metabolism to integrate nutritional and metabolic ecology
}

Thomas Ruiz, Apostolos-manuel Koussoroplis, Michael Danger, Jean-pierre Aguer, Nicole Morel-desrosiers, Alexandre Bec

\section{To cite this version:}

Thomas Ruiz, Apostolos-manuel Koussoroplis, Michael Danger, Jean-pierre Aguer, Nicole Moreldesrosiers, et al.. Quantifying the energetic cost of food quality constraints on resting metabolism to integrate nutritional and metabolic ecology. Ecology Letters, 2021, 24 (11), pp.2339-2349. 10.1111/ele.13855 . hal-03408463

\section{HAL Id: hal-03408463 \\ https://hal.science/hal-03408463}

Submitted on 29 Oct 2021

HAL is a multi-disciplinary open access archive for the deposit and dissemination of scientific research documents, whether they are published or not. The documents may come from teaching and research institutions in France or abroad, or from public or private research centers.
L'archive ouverte pluridisciplinaire HAL, est destinée au dépôt et à la diffusion de documents scientifiques de niveau recherche, publiés ou non, émanant des établissements d'enseignement et de recherche français ou étrangers, des laboratoires publics ou privés. 
Title:

Quantifying the energetic cost of food quality constraints on resting metabolism to

\section{Authors:}

6 Thomas RUIZ ${ }^{1}$ (thomas.ruiz@uca.fr),

7 Apostolos-Manuel KOUSSOROPLIS ${ }^{1}$ (A-Manuel.KOUSSOROPLIS@ @uca.fr),

8 Michael DANGER ${ }^{2}$ (michael.danger@univ-lorraine.fr),

9 Jean-Pierre AGUER ${ }^{1}$ (J-Pierre.AGUER@uca.fr),

10 Nicole MOREL-DESROSIERS ${ }^{1}$ (Nicole.MOREL@uca.fr),

11 Alexandre BEC ${ }^{1}$ alexandre.BEC@uca.fr $)$

Affiliations : ${ }^{1}$ Université Clermont Auvergne, CNRS, LMGE, F-63000 Clermont-Ferrand, France. ${ }^{2}$ Université de Lorraine, CNRS, LIEC, F-57000 Metz, France

Short title: Linking nutritional and metabolic ecology

Keywords: Metabolic ecology, Nutrition, Secondary production, Daphnia, stoichiometry, fatty acids, sterols

\section{Article Type: Letters}

20 Statement of authorship:

21 TR, AMK, MD and AB conceived the idea and developed the experimental protocol. TR ran the 22 experiment and acquired the data. TR, AMK and $\mathrm{AB}$ led the writing of the manuscript. All authors 23 contributed critically to the draft and gave the final approval for publication.

\section{Data accessibility statement:}

26 The data supporting the paper conclusions and $\mathrm{R}$ scripts associated are available on Figshare 27 (doi:10.6084/m9.figshare.13234277).

\section{Words in abstract: 150}

29 Words in main text: 4863

30 Number of references: 50

31 Number of figures: $3+$ Supplementary figures: 1

Number of Tables: $1+$ Supplementary tables: 1

Corresponding author: Thomas RUIZ; LMGE 1 Impasse Amélie Murat 63170 Aubière FRANCE; thomas.ruiz@uca.fr ; 0634194327 


\section{Abstract}

40 Consumer metabolism controls the energy uptake from the environment and its 41 allocation to biomass production. In natural ecosystems, available energy in food often

42 fails to predict biomass production which is also (co)limited by the relative availability of 43 various dietary compounds. To date, the link between energy metabolism and the effects 44 of food chemical composition on biomass production remains elusive. Here, we measured 45 the resting metabolic rate (RMR) of Daphnia magna along ontogeny when undergoing 46 various (non-energetic) nutritional constraints. All types of dietary (co)limitations (Fatty 47 acids, Sterols, Phosphorus) induced an increase in mass-specific RMR up to $128 \%$ between 48 highest and lowest quality diets. We highlight a strong negative correlation between RMR 49 and growth rate indicating RMR as a promising predictor of consumer growth rate. We 50 argue that quantifying the energetic cost imposed by food quality on individual RMR may 51 constitute a common currency enabling the integration of nutritional and metabolic 52 ecology. 
60 Introduction

Resting metabolic rate (RMR), sometimes reported as standard metabolic rate, is a

62 central component of individuals' energetics since it reflects the necessary cost of self-

63 maintenance subsequently constraining the energy allocation to other functions such as

64 growth or reproduction (McNab 2002). This key role made RMR a central component of

65 metabolic ecology from the Kleiber's power law (Kleiber 1932) through to the more recent

66 metabolic theory of ecology (MTE - (Brown et al. 2004)). MTE uses the allometric scaling

67 of RMR with body size and temperature to assess the rates at which organisms expend

68 (metabolism) and store energy as biomass (growth and reproduction). Thus, MTE

69 constitutes a quantitative approach that aims to predict the fluxes and stores of energy

70 between and within trophic levels (Brown et al. 2004). Yet, focusing on energy as sole

71 currency fails to explain the high spatial and temporal variability in energy transfer

72 efficiencies observed between trophic levels, particularly at the plant-animal interface

73 (Brett \& Goldman 1997).

74 To explain this variability, one needs to consider that energy transfer is coupled to

75 and driven by material transfer (Cebrian et al. 2009). Material can obviously be converted

76 into energy to fuel metabolism but is also required to build structure and ensure vital

77 functions (Clarke 2019). The non-substitutability of some essential compounds in

78 organism's physiology coupled with their inability to synthesize them de-novo require the

79 availability, at appropriate proportions, of such compounds in food resources. The disparity

80 between the consumer's nutritional demand for and the resources supply in essential

81 compounds -often referred to as food quality- drives the energy transfers between trophic 
82 levels via its effect on consumer growth and reproductive rates. Over the last decades,

83 identifying quantitative markers of food quality became an important task in ecological

84 research (Elser et al. 2000; Müller-Navarra et al. 2000; Ravet et al. 2012). For example, a

85 highly unsaturated fatty acid (HUFA) has been suggested to predict secondary production

86 in lakes (Müller-Navarra et al. 2000). At the same time, Elser et al (2000) showed that the

87 carbon $(\mathrm{C})$ to phosphorus $(\mathrm{P})$ ratio of food can also constrain herbivore growth efficiency.

88 Following these early works, the biochemical view of trophic interactions unveiled a great

89 variety of dietary compounds such as n-3 and n-6 polyunsaturated fatty acids (PUFA)

90 (Wacker \& von Elert 2001) and phytosterols (Martin-Creuzburg et al. 2006) as important

91 determinants of food quality limiting aquatic consumer growth either alone or in

92 combination (Sperfeld et al. 2012; Marzetz et al. 2017). The great variety of determinants

93 of food quality has split nutritional ecology into a patchwork of sub-disciplines which

94 focused on material transfer, often forsaking energy transfers of early works of trophic

95 ecology (Lindeman 1942). Several authors identify this segregation as an obstacle to our

96 understanding of ecosystem structure and functioning and call for the integration of these

97 two currencies into a common framework (Allen \& Gillooly 2009; Hillebrand et al. 2009;

98 Sperfeld et al. 2017; Barnes et al. 2018). We argue that the first step towards this goal is to

99 understand how limitation by material transfer (food quality) affects the way consumers

100 dissipate (RMR) and store energy (growth / reproduction).

101 In that aim, we used Daphnia magna to test whether and how individual RMR can

102 be affected by food quality. Besides allowing the exclusion of genetic differences of RMR,

103 the use of Daphnia magna is advantageous since the nutrition of this key aquatic organism

104 has been extensively studied over the last decades. Thanks to this rich literature we know 
105 that daphnids commonly face both stoichiometric (high food C:P) and biochemical (low

106 PUFA and/or sterols contents) dietary limitations in the field (Elser et al. 2000; Müller-

107 Navarra et al. 2004; Sperfeld et al. 2012). Here, we measured individual resting metabolic

108 rate (RMR) and juvenile growth rate (i.e., from birth to sexual maturity) along a gradient

109 of realistic food qualities incorporating various degrees of biochemical and stoichiometric

110 limitation, as well as their combination. We used a direct microcalorimetric method to

111 precisely measure how energy is dissipated by RMR (Ruiz et al. 2018). We hypothesized

112 that independently of its proximal cause (i.e. PUFA, Sterol or stoichiometry), sub-optimal

113 food quality may impose additional energetic costs to maintain organismal homeostasis,

114 ultimately inducing an increase of individual RMR. We argue that quantifying the energetic

115 cost imposed by food quality on individual RMR may constitute a common currency

116 further enabling the integration of food quality and metabolic ecology.

118 Materials and Methods

\section{$120 \quad$ Selection of Daphnia diets}

121 The quality of food for Daphnia is typically evaluated by measuring its effect on juvenile 122 growth rate (Wacker \& von Elert 2001; Martin-Creuzburg et al. 2006; Ravet et al. 2012)

123 in feeding experiments where the food quantity is unrestricted (eg above the incipient 124 limiting level, $\left.0.7 \mathrm{mgCL}^{-1}\right)$. Chlorophyte algae grown on P-limited medium are often used 125 to study stoichiometric limitations whereas different phytoplanktonic species are used in 126 light of their biochemical composition to assess limitations in sterol, polyunsaturated fatty 
127 acids (PUFA: 2 or 3 double bonds) and highly unsaturated fatty acids (HUFA: more than 1283 double bonds).

Here, our aim was to study the relationship between RMR and food quality as 130 reflected in Daphnia growth rate. To do so we created a food quality gradient based on 7 131 different diets for which nutritional quality was previously assessed in growth rate 132 experiments. These 7 treatments were used as training dataset to define the relation linking 133 RMR and growth rate. Besides the fact that these 7 diets could be ranked in terms of 134 expected growth rate they also differed in the proximate cause of growth limitation: i.e. 135 unbalanced stoichiometry, sterol, PUFA and HUFA limitation. To induce differences in 136 stoichiometric (P) balance we used the chlorophyte Chlamydomonas reinhardtii SAG 7781 137 grown either on P-replete (Chlamydomonas $\mathrm{P}+$ ) or P-limited medium (Chlamydomonas P138 ) (Ruiz et al. 2018). To create contrasts in biochemical nutritional constraints, we used 139 different phytoplankton strains: Cryptomonas sp. SAG 2680 (Cryptomonas P+), a 140 cryptophyte rich in sterols, PUFA and HUFA constituting an excellent quality food for 141 daphnids (Bec et al. 2006) and Chlamydomonas reinhardtii SAG 7781 (Chlamydomonas $142 \mathrm{P}+$ ) considered as a good quality food due to its high PUFA content (especially 18-3w3) 143 (Denoux et al. 2017). The lower food quality of chlorophytes when compared to 144 cryptophytes is mainly explained by the absence of the HUFA 20:5 $\omega 3$ (Martin-Creuzburg 145 \& von Elert 2009). Synechococcus sp. strain BO8809, a cyanobacteria of poor dietary 146 quality was used to induce HUFA, PUFA and sterol limitations of Daphnia growth (Bec 147 et al 2006). We gradually alleviated sterol limitation by supplementing Synechococcus $s p$. 148 with liposomes containing cholesterol (see below liposomes preparation) to create a 149 gradient of cholesterol availability with $2,4,8$ and $12 \mu \mathrm{g}$ cholesterol per $\mathrm{mg} \mathrm{C}$ in the final 
150 food suspension. In addition to the diets mentioned above, we further included 3 other diets

151 for which the food quality rank within our gradient (see above) is unknown: P-limited

152 Cryptomonas sp. SAG 2680 (Cryptomonas P-), a mixed diet composed of Chlamydomonas

153 reinhardtii SAG 7781 (representing 20\% of food C) and Synechococcus sp. strain BO8809

154 (representing $80 \%$ of food C) as well as a lyophilized sample of natural lake seston (i.e.

155 biotic and/or abiotic matter floating in the water column, here the fraction $<50 \mu \mathrm{m}$ ) sampled

156 during spring in a lake from the floodplain of the Allier river (France). These 3 dietary

157 treatments were considered as more complex diets since we have no a priori regarding

158 their consequences on consumer growth. They were thus used as test dataset to assess the

159 robustness of our regression with diets of unknown quality. All these dietary treatments are

160 summarized in Table 1.

161 All phytoplankton species were grown on a modified WC medium (Von Elert \& 162 Wolffrom 2001) with $7.1 \mathrm{mg} . \mathrm{L}^{-1}$ or $0.35 \mathrm{mg} . \mathrm{L}^{-1}$ phosphates at $20^{\circ} \mathrm{C}$ and under a permanent 163 artificial light flux of $120 \mu \mathrm{mol}$ quanta. $\mathrm{m}^{-2} \cdot \mathrm{s}^{-1}$. They were cultured semi-continuously at a 164 dilution rate of $0.2 \mathrm{~d}^{-1}$ in aerated (compressed sterile-filtrated air) $5 \mathrm{~L}$ vessels. Food 165 suspensions were prepared by centrifugation and resuspension of the cultured cells in 166 Volvic @ mineral water. The carbon concentrations of the food suspensions were estimated 167 from photometric light extinction $(800 \mathrm{~nm})$ and from carbon-extinction regressions 168 determined prior to the experiment.

\section{Experimental setup:}

We used the same clonal line of Daphnia magna (clone IRTA 2) in our experiment

171 to exclude any genotypic variability. The stock cultures of daphnids were kept in Volvic 
172 water@ at $20^{\circ} \mathrm{C}$ on a $14: 10 \mathrm{~h}$ day:night cycle and fed with Chlamydomonas reinhardtii (at

$1733 \mathrm{mgC}^{-1} \mathrm{~L}^{-1}$ ) for more than 3 generations before the beginning of the experiments. Due to the

174 fact we could only run 12 RMR measurements simultaneously (see below), the experiments

175 were run sequentially in 3 groups of 3 or 4 dietary treatments (Chlamydomonas $\mathrm{P}+$,

176 Chlamydomonas P-, Cryptomonas P+ / Cryptomonas P-, mixed diet, natural seston /

177 Synechococcus $s p+2-4-8-12 \mu \mathrm{g}$ cholesterol). The same temperature - light conditions

178 were kept for the experiments. To reduce inter-individual differences and permit

179 experiment reproducibility, all the daphnids used in the experiment were third-clutch

180 neonates (born within 8 hours) of the same clonal line (see details in appendix Figure S1).

181 During the first 24h, neonates were fed on a suspension of Chlamydomonas reinhardtii (3

$\left.182 \mathrm{mgC} . \mathrm{L}^{-1}\right)$ to ensure that all individuals reached a sufficient size to, later, perform

183 measurements of metabolic rate. After this first day, daphnids were randomly distributed

184 into 3 replicate jars for each dietary treatment with 12 daphnids per jar. Each of these jars

185 was filled with $500 \mathrm{~mL}$ Volvic $@$ water and with ad-libitum food suspensions $\left(3 \mathrm{mgC} . \mathrm{L}^{-1}\right)$.

186 This breeding medium was renewed daily to avoid any food quantity limitation and

187 crowding effects during all the experiment duration. Each day of measurement, one

188 individual was randomly selected in each replicate of each dietary treatment to determine

189 its RMR by microcalorimetry (see below). After measuring its RMR, the individual was

190 placed into a pre-weighed aluminum container, dried $48 \mathrm{~h}$ at $60^{\circ} \mathrm{C}$ and weighed (Sartorius;

191 ME36S; Goettingen-Germany). The dry weight reported here was used to define the

192 growth rate of daphnids as:

$$
G R=\frac{\ln (W t)-\ln (W 0)}{t} \quad E q .1
$$


194 Where GR is the growth rate, $\boldsymbol{W} \boldsymbol{t}$ is the weight (in $\mu \mathrm{g}$ ) at age $\boldsymbol{t}$ (in days) and $\boldsymbol{W} \boldsymbol{0}$ the weight 195 (in $\mu \mathrm{g}$ ) of neonates reflecting the mean dry weight of 3 pools of 15 individuals at birth $196(n=45)$.

197 In each treatment, the experiment was stopped when daphnids reached maturity (i.e. after 198 eggs deposition in the brood pouch) to avoid misleading measurement of metabolic rate 199 due to the development from eggs to juvenile in daphnids brood pouch. Mature organisms 200 were weighed as described above and the juvenile growth rates were calculated using 201 equation 1.

202

\section{Resting metabolic rate measurement by microcalorimetry:}

Resting metabolic rate (RMR) of daphnids was determined through the measurement of the heat produced by each individual using a TAM III microcalorimetric system of the heat-conduction type (Waters, TA Instruments; Newcastle, United States) enabling the continuous and simultaneous monitoring of 12 individuals. Before RMR measurement, daphnids were rinsed three times with Volvic $@$ water to remove all food particles and they were kept $1 \mathrm{~h}$ in clean water to exclude excretory products. They were then placed into specific disposable $4 \mathrm{~mL}$ ampoules containing $2 \mathrm{~mL}$ of Volvic $(\mathcal{O}$ water and $2 \mathrm{~mL}$ of air. The ampoules were then sealed and introduced in the 12 minicalorimeters. The data collection was performed with the TAM Assistant Software (Water, TA Instruments; Newcastle, United States): for each individual, a heat-flow value was collected every second during $8 \mathrm{~h}$. The last two hours of measurement (i.e. 6-8h after insertion) were used to define individual RMR, this method ensuring the stability of the heat-flow measurement and confirms that the RMR value excludes residual digestive activity (Ruiz et al. 2018) 
The 1-palmitoyl-2-oleoyl-phosphatidylglycerol (POPG, Sigma-Aldrich) and 1-

218 palmitoyl-2-oleoyl-phosphatidylcholin (POPC; Sigma-Aldrich) were dissolved in ethanol

219 at respective concentrations of 3 and $7 \mathrm{mg} \mathrm{mL}^{-1}$. In parallel, cholesterol was dissolved in

220 ethanol at a concentration of $20 \mathrm{mg} \cdot \mathrm{mL}^{-1}$. A mix containing $1 \mathrm{~mL}$ of POPG and POPC

221 solution plus $400 \mu \mathrm{L}$ of cholesterol solution was dried using a rotary evaporator and re-

222 suspended in $10 \mathrm{~mL}$ buffer $\left(20 \mathrm{mmol} . \mathrm{L}^{-1} \mathrm{NaPi}, 150 \mathrm{mmol} \cdot \mathrm{L}^{-1} \mathrm{NaCl}\right)$. This suspension was

223 then sonicated and centrifuged $\left(150000 \mathrm{~g}, 90 \mathrm{~min}, 4^{\circ} \mathrm{C}\right)$. The pellet was collected and

224 resuspended in buffer to remove free cholesterol from the liposome suspension (Martin-

225 Creuzburg et al. 2008; Denoux et al. 2017). Concentration of cholesterol was measured on

226 the final liposomes suspension used to supplement dietary treatment.

\section{Mineral and Biochemical analyses of food treatment:}

The mineral analyses were performed on suspensions of our phytoplankton cultures

229 adjusted to an absorbance of 0.8 at $800 \mathrm{~nm}$. These food suspensions were diluted 10 times 230 and their $\mathrm{C}$ content measured with a $\mathrm{CHN}$ analyser (NA-2100-Protein, ThermoQuest-CE

231 Instruments, Italy). Phosphorus content was estimated based on molybdenum blue reaction

232 after potassium persulfate oxidation (Ruiz et al. 2018) directly on the sample and on the 233 sample diluted 10 times. In parallel food suspensions with the similar concentration (i.e. 234 absorbance) were dried and weighted to report the $\mathrm{C}$ and $\mathrm{P}$ content per unit of dry mass. 235 Total lipids were extracted from food suspensions containing $1 \mathrm{mgC}$ using 236 chloroform/methanol (see Denoux et al. 2017 for further details). Lipids extract used to 237 fatty acid analysis were then submitted to an acid-catalysed transesterification producing 238 fatty acid methyl esters (Denoux et al. 2017). For sterol analysis, lipids extract undergone 
239 an alkaline hydrolysis (saponification) to specifically extract sterols. The fatty acid methyl

240 esters and sterols extracts were analysed by gas chromatography (6850-Network-GC,

241 Agilent-technologies, USA) (Martin-Creuzburg et al. 2006). Mineral and biochemical

242 composition of diets are given in Table $\mathbf{1 .}$

\section{Statistical analysis:}

244 The daphnids' juvenile growth rates were log transformed to meet ANOVA 245 assumptions. The differences between dietary treatments were tested using ANOVA 246 analysis followed by a post hoc Tukey test.

247 In order to estimate the ontogenetic mass-scaling exponent $(b)$ for each dietary 248 treatment, the RMR of daphnids was plotted against body mass and fitted to the following 249 linear model (Brown et al. 2004) for each dietary treatment separately using least squares 250 regression:

$$
\log (R M R)=\log (a)+b * \log (M) \quad E q .2
$$

252 where $\boldsymbol{a}$ is the proportionality constant and $\boldsymbol{b}$ the scaling exponent linking RMR to body 253 mass $(\boldsymbol{M})$. To check for the effect of replicate jar on our results we used the R package 254 "rstanarm" to fit a model including jar as random effect as:

$$
\log (R M R) \sim \log (\text { Mass }) * \text { Treatment }+(1 \mid \text { Jar }) \quad \text { Eq.3 }
$$

256 And a model excluding the effect of jars as:

$$
\log (\operatorname{RMR}) \sim \log (\text { Mass }) * \text { Treatment } \quad \text { Eq. } 4
$$

258 Equation 3 and 4 were run on the data including all dietary treatments. We used the leave259 one-out-cross validation (LOO) procedure (R package "loo") to test which model offered 260 a better fit to our data. LOO information Criterion (LOOIC) is appropriate to handle model 261 uncertainty and reduces the potential overfitting of models with more parameters. Here the 
262 model excluding "jar" (equation 4) appears as the top-ranked model since it exhibits an 263 expected $\log$ predictive density (ELPD) of 0 and $\triangle$ LOOIC of 1.2 with the model including 264 jars (equation 3) (Vehtari et al. 2017). We thus used the model excluding "jar" parameter 265 to test the effect of mass and dietary treatments on consumers RMR. In addition, we used 266 non-parametric bootstrapping with replacement $(n=10000)$ to estimate the uncertainty 267 around the $\boldsymbol{b}$ estimates for the different dietary treatments (Efron \& Tibshirani 1986). For 268 each bootstrap iteration, the $\boldsymbol{a}$ and $\boldsymbol{b}$ estimates for each dietary treatments were also used 269 to predict the mass-specific RMR for individuals of $100 \mu \mathrm{g}$ (MS-RMR 100$)$. Significant 270 differences in bootstrapped $\boldsymbol{b}$ and MS-RMR 100 estimates between dietary treatments were 271 concluded in the lack of overlap between their 95\%CI.

272 In order to evaluate the ability of RMR metrics to predict food quality, we constructed 273 linear models relating the mass-specific RMR measurements at a given age (5 days old;

$274 \mathrm{MS}^{-R M R_{5 d}}$ ), MS-RMR 100 estimates and $\boldsymbol{b}$ estimates to log-transformed growth rate using 275 the training dataset $(\mathrm{N}=21$, see above). We then used these models on the above-mentioned 276 RMR metrics measured in the test dataset $(\mathrm{N}=9)$ to predict the growth rates. The goodness 277 of the predictions was evaluated by regressing the observed (in the test dataset) to predicted 278 values (Piñeiro et al. 2008).

279

$280 \quad \underline{\text { Results }}$

281 Effects of dietary treatments on growth:

282 The differences in availability of essential biochemical and mineral dietary compounds in 283 our treatments significantly affected individuals' growth rate (ANOVA, $\mathrm{F}_{(9,110)}=9.94$; $284 \mathrm{p}<0.001$, Figure 1.B). Individuals exhibited the lowest growth rate of $0.32 \mathrm{~d}^{-1}$ when fed 
285 with Synechococcus sp. supplemented with $2 \mu \mathrm{g} \cdot \mathrm{mgC}^{-1}$ cholesterol. The supplementation 286 of Synechococcus sp. with cholesterol concentrations up to $12 \mu \mathrm{g} \cdot \mathrm{mgC}^{-1}$ alone significantly 287 increased individual's growth rate up to $0.41 \mathrm{~d}^{-1}$ (Tukey post-hoc test versus Synechococcus $288 s p .+2 \mu$ g.mgC cholesterol: $\mathrm{p}<0.001)$. Individual's growth rate further increased to $0.5 \mathrm{~d}^{-1}$ 289 when fed with Chlamydomonas P+, which contains PUFAs, (Tukey post-hoc test versus 290 Synechococcus sp. $+12 \mu \mathrm{g} . \mathrm{mgC}$ cholesterol: $\mathrm{p}<0.001)$ ) and reached a maximum value of $2910.64 \mathrm{~d}^{-1}$ when both PUFAs and HUFAs were available on a diet composed of Cryptomonas $292 \mathrm{P}+$ (Tukey post-hoc test versus Chlamydomonas $\mathrm{P}+\mathrm{p}<\mathrm{p}$.001). From a stoichiometric 293 viewpoint, the deficiency of nutrient (phosphorus) significantly reduced individuals growth 294 rate in Cryptomonas sp. treatments (growth declines from $0.64 \mathrm{~d}^{-1}(\mathrm{P}+)$ to $0.49 \mathrm{~d}^{-1}(\mathrm{P}-)$, 295 Tukey post-hoc test, $\mathrm{p}<0.001$ ) but not in Chlamydomonas reinhardtii treatments (growth 296 declines from $0.5 \mathrm{~d}^{-1}(\mathrm{P}+)$ to $0.45 \mathrm{~d}^{-1}(\mathrm{P}-)$, Tukey post-hoc test, $\left.\mathrm{p}=0.06\right)$. Individuals fed with 297 mixed diet and natural seston exhibited growth rates of $0.38 \mathrm{~d}^{-1}$ and $0.39 \mathrm{~d}^{-1}$ respectively, 298 which correspond to the lower range of growth rates reported in this study (Figure 1.A-B).

Effects of dietary treatments on resting metabolic rate:

The progressive increase in availability of essential biochemical compounds 301 induced significant decrease of individuals resting metabolic rate (Figure 1.C-E). The 302 worst dietary treatment (Synechococcus sp. with $2 \mu \mathrm{g} \cdot \mathrm{mgC}^{-1}$ cholesterol) resulted in the 303 highest MS-RMR 100 of $0.048 \mu \mathrm{W} . \mu \mathrm{g}^{-1}(95 \% \mathrm{CI}[0.047 ; 0.05])$ (Figure 1.E). The successive 304 supplementations of Synechococcus sp. with cholesterol (from $2 \mu \mathrm{g} \cdot \mathrm{mgC}^{-1}$ to $12 \mu \mathrm{g} \cdot \mathrm{mgC}^{-1}$ ) 305 gradually alleviated the sterol deficiency with the consequence of decreasing the mass306 specific MS-RMR 100 down to $0.035 \mu \mathrm{W} \cdot \mu \mathrm{g}^{-1}$ (95\%CI [0.034; 0.036]). The MS-RMR 100 307 reached values of $0.029 \mu \mathrm{W} . \mu \mathrm{g}^{-1}(95 \% \mathrm{CI}[0.028$; 0.03]) when PUFAs were available and 
$3080.021 \mu \mathrm{W} \cdot \mu \mathrm{g}^{-1}(95 \% \mathrm{CI}[0.021 ; 0.022])$ when both PUFA and HUFA were available.

309 Deficiency of phosphorus also significantly increased individuals MS-RMR 100 which 310 shifted from $0.029 \mu \mathrm{W} . \mu \mathrm{g}^{-1}(95 \% \mathrm{CI}[0.028 ; 0.03])$ to $0.034 \mu \mathrm{W} . \mu \mathrm{g}^{-1}(95 \% \mathrm{CI}[0.031 ; 0.036])$

311 between Chlamydomonas $\mathrm{P}+$ and Chlamydomonas $\mathrm{P}$ - treatments respectively. In a 312 comparable fashion to what was reported for Chlamydomonas, the $\mathrm{P}$ limitation in 313 Cryptomonas shifted the MS-RMR 100 from $0.021 \mu \mathrm{W} . \mu \mathrm{g}^{-1}(95 \% \mathrm{CI}[0.021 ; 0.022])(\mathrm{P}+)$ to $3140.029 \mu \mathrm{W} . \mu \mathrm{g}^{-1}(95 \% \mathrm{CI}[0.028 ; 0.03])(\mathrm{P}-)$. The two other diets from the test dataset 315 exhibited intermediate values with $0.042 \mu \mathrm{W} \cdot \mu \mathrm{g}^{-1}(95 \% \mathrm{CI}[0.04 ; 0.045])$ for the mixed diet 316 and $0.036 \mu \mathrm{W} . \mu \mathrm{g}^{-1}(95 \% \mathrm{CI}[0.035 ; 0.037])$ for the natural seston.

\section{Effects of dietary treatments on metabolic scaling during ontogeny:}

318 We also reported a significant variation of the RMR-mass scaling during ontogeny 319 in response to dietary quality. Here, the worst diet composed of Synechococcus sp. with $3202 \mu \mathrm{g} \cdot \mathrm{mgC}^{-1}$ cholesterol exhibited a scaling exponent (coefficient $\boldsymbol{b}$ ) of 1 (95\% CI [0.98; 321 1.02]) which is the highest value reported. The supplementations of Synechococcus sp. 322 with cholesterol significantly reduced the value of $\boldsymbol{b}$ which, however, remained close to 1 323 when $4 \mu \mathrm{g} \cdot \mathrm{mgC}^{-1}(0.9 ; 95 \%$ CI $[0.88 ; 0.92])$ and $8 \mu \mathrm{g} \cdot \mathrm{mgC}^{-1}(0.9 ; 95 \% \mathrm{CI}[0.89 ; 0.92])$ 324 cholesterol were added. In Synechococcus sp. supplemented with $12 \mu \mathrm{g} \cdot \mathrm{mgC}^{-1}$ cholesterol 325 the value of $b$ reached 0.79 (95\% CI $[0.76 ; 0.81])$. The treatments composed of 326 Chlamydomonas P+ which contains PUFA exhibited $\boldsymbol{b}$ value of 0.67 (95\%CI [0.65; 0.69]). 327 The lowest value for $b$ was 0.46 (95\% CI $[0.44 ; 0.48])$ and was noted for daphnids fed on 328 Cryptomonas $\mathrm{P}+$ (Figure 1.D). 
Our linear models linking growth rate to MS-RMR fit well with the data both for 331 MS-RMR 5 d $\left(\mathrm{F}_{(1: 19)}=167.9\right.$, p-value $\left.<0.001, \mathrm{R}^{2}=0.92\right)$ and $\mathrm{MS}-\mathrm{RMR}_{100}\left(\mathrm{~F}_{(1: 19)}=456.6\right.$, p332 value $<0.001, \mathrm{R}^{2}=0.96$ ) (Figure 2). Similarly, our linear model linking growth rate and 333 RMR scaling exponent $(\boldsymbol{b})$ also fits well with our data $\left(\mathrm{F}_{(1: 19)}=506.9, \mathrm{p}\right.$-value $<0.001$, $334 \mathrm{R}^{2}$ :0.96) (Figure 2). The high correlation between RMR and growth reported here when 335 all dietary treatments of our training dataset are included highlights a strong correlation 336 between RMR and growth rate regardless of the nature of the nutritional constraint.

\section{Growth rate predictions from individuals RMR:}

338 Using the linear model drawn above we predicted individual growth rate on the test 339 dataset from MS-RMR 5 d, MS-RMR 100 and $\boldsymbol{b}$. The scaling exponent $(b)$ appeared as a good 340 predictor of individual growth since the "observed versus predicted" linear regression 341 exhibits a slope of 0.97 (CI95\% [0.61; 1.32]), an intercept of 0.01 (CI95\%[-0.14, 0.16]) 342 and a high $\mathrm{R}^{2}(0.80)$. The $\mathrm{MS}-\mathrm{RMR}_{100}$ also provide useful prediction of individual growth 343 (slope 0.81 (CI95\%[0.45;1.16]); intercept: 0.06 (CI95\%[-0.09; 0.23]) and R²:0.74). The 344 MS-RMR5d was the less useful predictor of growth rate as reported by the low $\mathrm{R}^{2}(0.43)$ 345 and the uncertainty in slope $(1.25$ (CI95\%[0.08; 2.42])) and intercept $(-0.14$ (CI95\%[-0.71; $3460.41])$ ). Note that these regressions were based on a small number of datapoints (3 347 treatments $\times 3$ replicates $=9$ ) which explains the large confidence intervals in the regression 348 parameter estimates. 
Here we found that regardless of their nature (biochemical and/or elemental 353 constraint) food quality constraints systematically increase RMR. Between some dietary 354 treatments, the difference in RMR at a given mass $(100 \mu \mathrm{g})$ can be as large as $128 \%$ which 355 is comparable to the effect on RMR caused by a mass difference of one order of magnitude 356 (assuming $b=0.75$ (Brown et al. 2004)). We found a tight negative log-linear relationship 357 between mass-specific resting metabolic rate (MS-RMR $\left.{ }_{100}\right)$ and growth rate of Daphnia 358 magna submitted to various diets of contrasting quality. This relationship predicts 359 relatively well the growth rate from $M S-R_{100}$ in the complex diets of unknown food 360 quality used as test dataset (Natural seston, Mixed diet, Cryptomonas P-).

Due to its deficiency in sterols, polyunsaturated fatty acids (PUFA) and highly unsaturated fatty acids (HUFA) the cyanobacterium Synechococcus sp. is known to be of 363 very low dietary quality for Daphnia (Martin-Creuzburg \& von Elert 2009). The diet 364 composed of Synechococcus sp. with the lowest supplementation of cholesterol induced 365 the lowest growth rate and the highest MS-RMR 100 in this study. From a biochemical 366 viewpoint, the stepwise supply of sterols, PUFA (Chlamydomonas reinhardtii) and HUFA 367 (Cryptomonas sp.) gradually improved food quality (as reflected by the increase of juvenile 368 growth rate) inducing a decrease of individual MS-RMR 100 . As predicted by ecological 369 stoichiometry (Elser et al. 2000), the increasing availability of P in Chlamydomonas $\mathrm{P}+$ 370 (i.e. the reduction of dietary C:P) enhanced food quality and slightly decreased MS371 RMR $_{100}$ in agreement with Ruiz et al (2018). Phosphorus limitation had a much stronger 372 effect on Daphnia growth and MS-RMR 100 when fed on Cryptomonas sp. which contains 373 HUFA. Despite its high HUFA content, P-limited Cryptomonas sp. (Cryptomonas P-) did 374 not improve growth relative to both Chlamydomonas $\mathrm{P}+$ and Chlamydomonas $\mathrm{P}-$. This 
375 suggests that Daphnia fed on P-limited chlorophytes (Boersma 2000) are actually co-

376 limited by $\mathrm{P}$ and HUFA supply. Interestingly, this also indicates that the relationship

377 between MS-RMR 100 and food quality likely holds for situations where multiple nutrients

378 co-limit growth.

379 Taking a stoichiometric perspective, dietary limitations can be viewed as an 380 imbalance leading to excess in the supply of a nutrient relative to another (limiting)

381 nutrient. To maintain internal homeostasis, consumers facing P limitation should actively 382 release excess C through respiration and/or excretion (Darchambeau et al. 2003; Jeyasingh 383 2007) resulting in a higher RMR (Ruiz et al. 2018). In this case, the excess carbon is lost 384 to growth and the increased RMR would reflect the cost of strict stoichiometric 385 homeostasis. In the same way, a biochemical limitation could be seen as an imbalance in 386 essential lipids supply (PUFA, HUFA, sterols) relative to other lipids. In this case too, 387 consumers might need to actively dispose lipids in excess and/or synthesize the required 388 compounds from their precursors (e.g. HUFA from PUFA) in order to maintain membrane 389 homeostasis. Yet, the lipid composition of cell membranes is not strictly homeostatic and 390 depends to a certain extent on dietary supply (Clamp et al. 1997). Such diet-induced 391 modifications in membrane composition can affect the efficiency of membrane transport 392 proteins (NaKATPase, Ca2+ATPase,...) (Pepe et al. 1999) and overall mitochondrial 393 energetic efficiency (Stanley et al. 2012). Given that these transporters consume nearly 394 half of the energy (ATP) used by resting organisms (Rolfe \& Brown 1997) any decrease in 395 their efficiency could imply an energy expense that contributes to the increase of RMR we 396 observe in the case of dietary lipid limitation. 
Our results also highlight important dietary-induced differences in the ontogenetic

398 RMR-mass scaling coefficient $(b)$. This scaling coefficient is a central component of the 399 metabolic theory of ecology (Brown et al. 2004) or ontogenetic growth models (West et 400 al. 2001) and has long been assumed constant across the entire animal reign (Savage et al. 401 2004). Yet, over the last decade, the universality of this scaling coefficient has largely been 402 questioned (Glazier 2006; Burton et al. 2011). Here we show that food quality alone can 403 induce variations of this coefficient ranging from 1 (95\% CI [0.98; 1.02]) (Poor diet 404 Synechococcus sp. $+2 \mu \mathrm{g} \cdot \mathrm{mgC}^{-1}$ cholesterol) to 0.46 (95\% CI $\left.[0.44 ; 0.48]\right)$ (Rich diet 405 Cryptomonas $\mathrm{P}+$ ) which is as much as the entire range of interspecific variation in mass 406 scaling exponents reported in literature (White \& Seymour 2003; Glazier 2006). Our data 407 shows strong linear relationship between $b$ and growth rate. In Daphnia, juvenile growth 408 rate is usually tightly correlated to fecundity (Martin-Creuzburg et al. 2009). Hence, the 409 observed differences in $b$ likely reflect shifts in the temporal dynamic of the ratio between 410 structural and reproductive tissues along ontogeny. At the onset of sexual maturity, 411 Daphnia incorporates reproductive tissues (ovaries/eggs) in addition to the structural tissue 412 that constitutes its body mass. Compared to structural tissues, the production and 413 maintenance of reproductive tissues require considerably less energy per unit mass 414 (Kooijman 2009). Hence, the increasing contribution of reproductive tissues to body mass 415 during ontogeny gradually decreases the whole-body mass-specific energy requirements 416 ultimately reducing the scaling of RMR to body mass (coefficient $b$ ). While fecundity was 417 not measured here, several lines of published evidence support this hypothesis. In the 418 dietary treatments composed of Synechococcus sp. solely supplemented with cholesterol, 419 reproduction should not be possible or very reduced in Daphnia (Martin-Creuzburg et al. 
420 2006; Martin-Creuzburg \& von Elert 2009; Ravet et al. 2012). In our experiments, Daphnia

421 fed Synechococcus sp. $+2 \mu \mathrm{g} \cdot \mathrm{mgC}^{-1}$ cholesterol exhibits a nearly isometric RMR-mass

422 scaling $(b=1)$ which is in agreement with what is expected if the observed ponderal growth

423 would only be due to an increase of structural tissue (Glazier 2006). To be able to reproduce

424 Daphnia ideally requires HUFA, or at least their PUFA precursors (Martin-Creuzburg \& 425 von Elert 2009) at the cost of reduced reproductive output however. Hence, 426 Chlamydomonas-based diets which contain PUFA should enable reproduction yet to a 427 lesser degree compared to the HUFA-rich Cryptomonas sp. which usually maximizes 428 fecundity in Daphnia (Müller-Navarra et al. 2004; Bec et al. 2006; Denoux et al. 2017).

429 The difference between the $b$ estimates for the two treatments found here (Chlamydomonas 430 P+: 0.65, Cryptomonas P+: 0.46) could therefore reflect their different reproductive 431 outputs.

Overall, our results show that nutritional constraints cause a rise of RMR that is

433 proportional to the intensity of the constraint regardless of its nature (stoichiometry, PUFA, 434 sterols, co-limitation). Resting metabolic rate has been proposed as a transversal currency 435 underpinning ecological processes from individuals to the biosphere (Brown et al. 2004), 436 suggesting that our conclusions could be transposable to other taxa. By measuring the 437 dissipation of energy associated with food quality, regardless of whether one considers 438 MS-RMR dd $_{5}$, MS-RMR ${ }_{100}$ or RMR mass-scaling (b), our study shows that RMR can 439 quantify the energetic cost imposed by food quality constraints. The fact that complex diets 440 such as natural seston fall within the regression obtained from our food quality gradient 441 suggests that it might be possible to develop an instantaneous marker of food quality in 442 natural systems based on energy dissipation by RMR. For example, if food quality 
443 fluctuates at longer temporal scales than consumers developmental time, estimating

444 ontogenetic $b$ from coexisting cohorts of differently-sized individuals could provide a

445 proxy for food quality constraints on growth rate. Furthermore, quantifying diverse food

446 quality constraints in a unique energetic currency may also facilitate the integration of food

447 quality into metabolic theories. For example, Dynamic Energy Budget (DEB) theory offers

448 the possibility to explicitly model the flows of each limiting dietary compounds into the

449 organism (Kooijman 2009; Koussoroplis et al. 2019). Yet, this level of detail comes at the

450 cost of a high complexity and specificity as well as a large number of independent

451 parameters. In this regard, Sperfeld et al. (2017) stress the need to maintain DEB models

452 as simple as possible. Our results suggest that integrating food-quality dependence in the

453 DEB model parameters that account for energy dissipation (e.g. structural maintenance)

454 could be a simplified alternative to multi-reserve models.

455 Under pressure from global changes such as warming and eutrophication, food

456 quality in aquatic ecosystems is expected to decline as phytoplanktonic communities

457 become increasingly dominated by chlorophytes and cyanobacteria (Freeman et al. 2020).

458 Higher water temperature (Schulte 2015) and lower food quality -as we show here- lead to

459 increases of individual RMR that reflect a rising oxygen demand. Yet, warming and

460 eutrophication also lead to a decrease in water oxygen availability (Thomas et al. 2019;

461 Sokolova 2021). Hence, the combination of high temperature, low food quality and low

462 oxygen might exacerbate the risk of hypoxia for aquatic animals. In the same manner, poor

463 food quality might also interact with other stressors known to affect RMR such as food

464 quantity (Auer et al. 2015), pollutants (Rowe 1998) or predation stress (Glazier et al. 2020). 
Focus on the bioenergetic consequences of poor food quality stress enables its integration into a multiple stressors framework based on a common energetic currency.

467

468

469

470

471

472

473

474

475

476

477

478

479

480

481

482

483

484

485

486

487

488

489

490

491

492

493

494

495

496

497

498

499

500

\section{Acknowledgments}

Research project "PASSCAL" funded by I-site Clermont under the "Emergence" initiative for the CAP 20-25 (awarded to AB). We thank Neil Metcalfe and two anonymous reviewers for their insightful comments that helped improve this manuscript. We also thank Franck Jabot for his thoughtful reading and comments on a very first version of this manuscript.

\section{References}

Allen, A.P. \& Gillooly, J.F. (2009). Towards an integration of ecological stoichiometry and the metabolic theory of ecology to better understand nutrient cycling. Ecol. Lett., 12, 369384.

Auer, S.K., Salin, K., Rudolf, A.M., Anderson, G.J. \& Metcalfe, N.B. (2015). Flexibility in metabolic rate confers a growth advantage under changing food availability. J. Anim. Ecol., 84, 1405-1411.

Barnes, A.D., Jochum, M., Lefcheck, J.S., Eisenhauer, N., Scherber, C., O’Connor, M.I., et al. (2018). Energy Flux: The Link between Multitrophic Biodiversity and Ecosystem Functioning. Trends Ecol. Evol., 33, 186-197.

Bec, A., Martin-Creuzburg, D. \& von Elert, E. (2006). Trophic upgrading of autotrophic picoplankton by the heterotrophic nanoflagellate Paraphysomonas sp. Limnol. Oceanogr., 51, 1699-1707.

Boersma, M. (2000). The nutritional quality of P-limited algae for Daphnia. Limnol. Oceanogr., 45, 1157-1161.

Brett, M.T. \& Goldman, C.R. (1997). Consumer Versus Resource Control in Freshwater Pelagic Food Webs. Science, 275, 384-386.

Brown, J.H., Gillooly, J.F., Allen, A.P., Savage, V.M. \& West, G.B. (2004). TOWARD A METABOLIC THEORY OF ECOLOGY. Ecology, 85, 1771-1789.

Burton, T., Killen, S.S., Armstrong, J.D. \& Metcalfe, N.B. (2011). What causes intraspecific variation in resting metabolic rate and what are its ecological consequences? Proc. $R$. Soc. B Biol. Sci., 278, 3465-3473.

Cebrian, J., Shurin, J.B., Borer, E.T., Cardinale, B.J., Ngai, J.T., Smith, M.D., et al. (2009). Producer Nutritional Quality Controls Ecosystem Trophic Structure. PLOS ONE, 4, e4929. 
Clamp, A.G., Ladha, S., Clark, D.C., Grimble, R.F. \& Lund, E.K. (1997). The influence of dietary lipids on the composition and membrane fluidity of rat hepatocyte plasma membrane. Lipids, 32, 179-184.

Clarke, A. (2019). Energy Flow in Growth and Production. Trends Ecol. Evol., 34, 502-509.

Darchambeau, F., Faervig, P.J. \& Hessen, D.O. (2003). How Daphnia copes with excess carbon in its food. Oecologia, 136, 336-346.

Denoux, C., Martin-Creuzburg, D., Koussoroplis, A.-M., Perriere, F., Desvillettes, C., Bourdier, G., et al. (2017). Phospholipid-bound eicosapentaenoic acid (EPA) supports higher fecundity than free EPA in Daphnia magna. J. Plankton Res., 39, 843-848.

Efron, B. \& Tibshirani, R. (1986). Bootstrap Methods for Standard Errors, Confidence Intervals, and Other Measures of Statistical Accuracy. Stat. Sci., 1, 54-75.

Elser, J.J., Fagan, W.F., Denno, R.F., Dobberfuhl, D.R., Folarin, A., Huberty, A., et al. (2000). Nutritional constraints in terrestrial and freshwater food webs. Nature, 408, 578-580.

Freeman, E.C., Creed, I.F., Jones, B. \& Bergström, A. (2020). Global changes may be promoting a rise in select cyanobacteria in nutrient-poor northern lakes. Glob. Change Biol., 26, 4966-4987.

Glazier, D.S. (2006). The 3/4-Power Law Is Not Universal: Evolution of Isometric, Ontogenetic Metabolic Scaling in Pelagic Animals. BioScience, 56, 325.

Glazier, D.S., Borrelli, J.J. \& Hoffman, C.L. (2020). Effects of Fish Predators on the Mass-Related Energetics of a Keystone Freshwater Crustacean. Biology, 9, 40.

Hillebrand, H., Borer, E.T., Bracken, M.E.S., Cardinale, B.J., Cebrian, J., Cleland, E.E., et al. (2009). Herbivore metabolism and stoichiometry each constrain herbivory at different organizational scales across ecosystems. Ecol. Lett., 12, 516-527.

Jeyasingh, P.D. (2007). Plasticity in metabolic allometry: the role of dietary stoichiometry. Ecol. Lett., 10, 282-289.

Kleiber, M. (1932). Body size and metabolism. Hilgardia, 6, 315-353.

Kooijman, B. (2009). Dynamic Energy Budget Theory for Metabolic Organisation. 3rd edn. Cambridge University Press, Cambridge.

Koussoroplis, A., Schälicke, S., Raatz, M., Bach, M. \& Wacker, A. (2019). Feeding in the frequency domain: coarser-grained environments increase consumer sensitivity to resource variability, covariance and phase. Ecol. Lett., 22, 1104-1114.

Lindeman, R.L. (1942). The Trophic-Dynamic Aspect of Ecology. Ecology, 23, 399-417.

Martin-Creuzburg, D., Bec, A. \& von Elert, E. (2006). Supplementation with Sterols Improves Food Quality of a Ciliate for Daphnia magna. Protist, 157, 477-486.

Martin-Creuzburg, D. \& von Elert, E. (2009). Good food versus bad food: the role of sterols and polyunsaturated fatty acids in determining growth and reproduction of Daphnia magna. Aquat. Ecol., 43, 943-950.

Martin-Creuzburg, D., von Elert, E. \& Hoffmann, K.H. (2008). Nutritional constraints at the cyanobacteria- Daphnia magna interface: The role of sterols. Limnol. Oceanogr., 53, 456-468.

Martin-Creuzburg, D., Sperfeld, E. \& Wacker, A. (2009). Colimitation of a freshwater herbivore by sterols and polyunsaturated fatty acids. Proc. R. Soc. B Biol. Sci., 276, 1805-1814.

Marzetz, V., Koussoroplis, A.-M., Martin-Creuzburg, D., Striebel, M. \& Wacker, A. (2017). Linking primary producer diversity and food quality effects on herbivores: A biochemical perspective. Sci. Rep., 7, 1-9.

McNab, B.K. (2002). The physiological ecology of vertebrates: a view from energetics. Cornell University Press. New York. 
Müller-Navarra, D.C., Brett, M.T., Liston, A.M. \& Goldman, C.R. (2000). A highly unsaturated fatty acid predicts carbon transfer between primary producers and consumers. Nature, 403, 74-77.

Müller-Navarra, D.C., Brett, M.T., Park, S., Chandra, S., Ballantyne, A.P., Zorita, E., et al. (2004). Unsaturated fatty acid content in seston and tropho-dynamic coupling in lakes. Nature, 427, 69-72.

Pepe, S., Tsuchiya, N., Lakatta, E.G. \& Hansford, R.G. (1999). PUFA and aging modulate cardiac mitochondrial membrane lipid composition and $\mathrm{Ca}^{2+}$ activation of PDH. Am. J. Physiol.Heart Circ. Physiol., 276, H149-H158.

Piñeiro, G., Perelman, S., Guerschman, J.P. \& Paruelo, J.M. (2008). How to evaluate models: Observed vs. predicted or predicted vs. observed? Ecol. Model., 216, 316-322.

Ravet, J., Persson, J. \& Brett, M.T. (2012). Threshold dietary polyunsaturated fatty acid concentrations for Daphnia pulex growth and reproduction. Inland Waters, 2, 199-209.

Rolfe, D.F. \& Brown, G.C. (1997). Cellular energy utilization and molecular origin of standard metabolic rate in mammals. Physiol. Rev., 77, 731-758.

Rowe, C.L. (1998). Elevated standard metabolic rate in a freshwater shrimp (Palaemonetes paludosus) exposed to trace element-rich coal combustion waste. Comp. Biochem. Physiol. A. Mol. Integr. Physiol., 121, 299-304.

Ruiz, T., Bec, A., Danger, M., Koussoroplis, A.-M., Aguer, J.-P., Morel, J.-P., et al. (2018). A microcalorimetric approach for investigating stoichiometric constraints on the standard metabolic rate of a small invertebrate. Ecol. Lett., 21, 1714-1722.

Savage, V.M., Gillooly, J.F., Woodruff, W.H., West, G.B., Allen, A.P., Enquist, B.J., et al. (2004). The predominance of quarter-power scaling in biology. Funct. Ecol., 18, 257-282.

Schulte, P.M. (2015). The effects of temperature on aerobic metabolism: towards a mechanistic understanding of the responses of ectotherms to a changing environment. J. Exp. Biol., 218, 1856-1866.

Sokolova, I. (2021). Bioenergetics in environmental adaptation and stress tolerance of aquatic ectotherms: linking physiology and ecology in a multi-stressor landscape. J. Exp. Biol., 224, jeb236802.

Sperfeld, E., Martin-Creuzburg, D. \& Wacker, A. (2012). Multiple resource limitation theory applied to herbivorous consumers: Liebig's minimum rule vs. interaclive co-limitation. Ecol. Lett., 15, 142-150.

Sperfeld, E., Wagner, N.D., Halvorson, H.M., Malishev, M. \& Raubenheimer, D. (2017). Bridging Ecological Stoichiometry and Nutritional Geometry with homeostasis concepts and integrative models of organism nutrition. Funct. Ecol., 31, 286-296.

Stanley, W.C., Khairallah, R.J. \& Dabkowski, E.R. (2012). Update on lipids and mitochondrial function: impact of dietary n-3 polyunsaturated fatty acids. Curr. Opin. Clin. Nutr. Metab. Care, 15, 122-126.

Thomas, Y., Flye-Sainte-Marie, J., Chabot, D., Aguirre-Velarde, A., Marques, G.M. \& Pecquerie, L. (2019). Effects of hypoxia on metabolic functions in marine organisms: Observed patterns and modelling assumptions within the context of Dynamic Energy Budget (DEB) theory. J. Sea Res., 143, 231-242.

Vehtari, A., Gelman, A. \& Gabry, J. (2017). Practical Bayesian model evaluation using leave-oneout cross-validation and WAIC. Stat. Comput., 27, 1413-1432.

Von Elert, E. \& Wolffrom, T. (2001). Supplementation of cyanobacterial food with polyunsaturated fatty acids does not improve growth of Daphnia. Limnol. Oceanogr., 46, 1552-1558. 
595 Wacker, A. \& von Elert, E. (2001). Polyunsaturated fatty acids: Evidence for non-substitutable 596 biochemical resources in Daphnia galeata. Ecology, 82, 2507-2520.

597 West, G.B., Brown, J.H. \& Enquist, B.J. (2001). A general model for ontogenetic growth. Nature, $598 \quad 413,628-631$.

599 White, C.R. \& Seymour, R.S. (2003). Mammalian basal metabolic rate is proportional to body 600 mass2/3. Proc. Natl. Acad. Sci., 100, 4046-4049.

601 

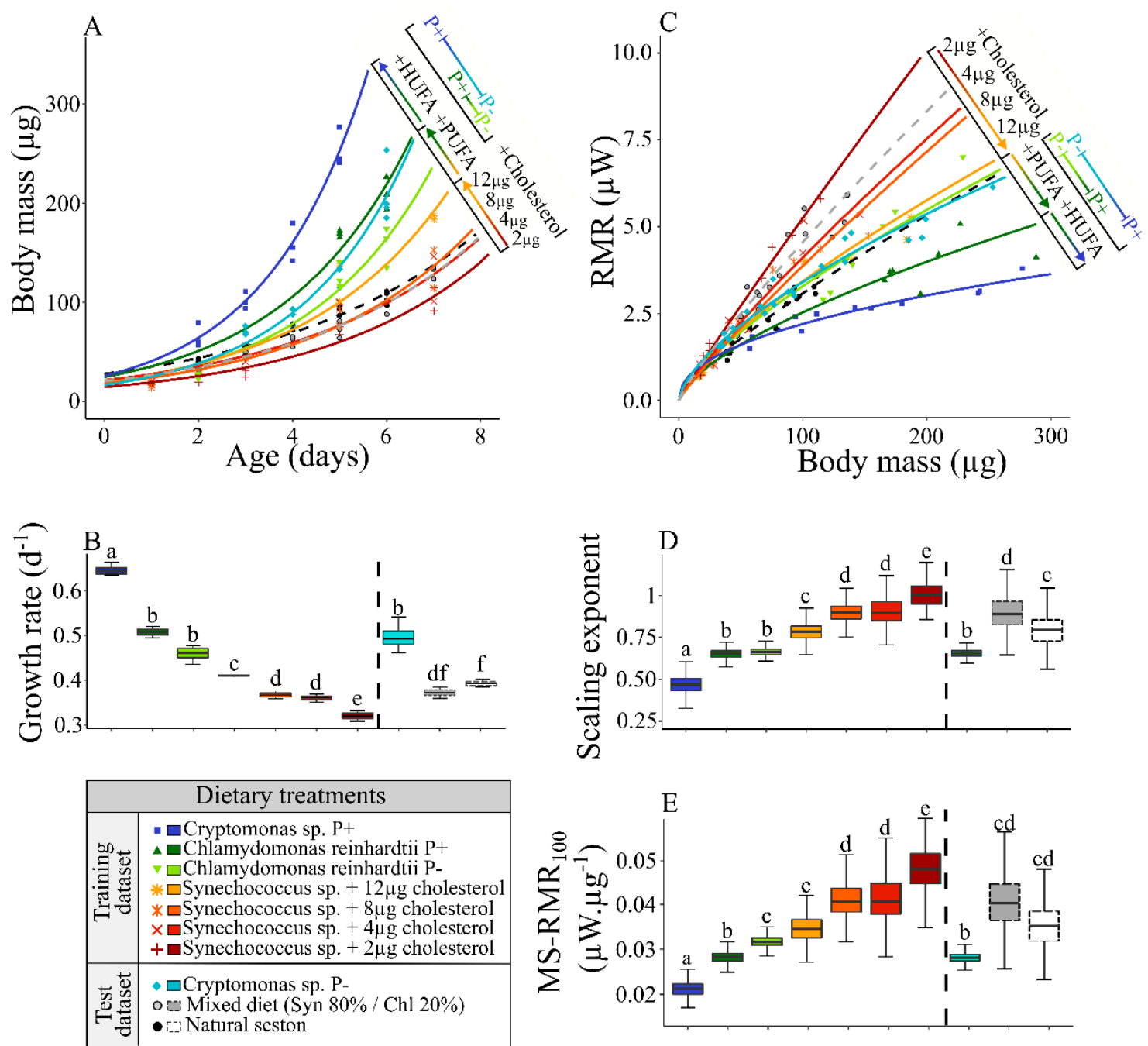

604

605

606

607

608

609

610

611

612

613

614

615

616
Figure 1. Individual growth and resting metabolic rate measured along dietary gradient. Left side panels show the evolution of individuals body mass versus age depending on dietary treatments $(A)$ and the corresponding juvenile growth rate $(B)$. Right side panels show the resting metabolic rate $(R M R)$ of individuals depending on their body mass and diet $(C)$, the coefficients defining the RMR scaling $(D)$ and the mass-specific RMR $(M S-R M R)$ of an individual of $100 \mu g(E)$. Values for panel $D$ and $E$ were predicted from log-linear regression between RMR and body mass. In each panel, colors represent dietary treatments. In panel $A$ and $C$ the dietary gradient exhibits the main limiting dietary compounds. Boxes represent interquartile ranges and letters are significant differences based on post-hoc Tukey test (panel B) or based on 95\% confidence intervals (panel D \& E, see Table S1 for details). 

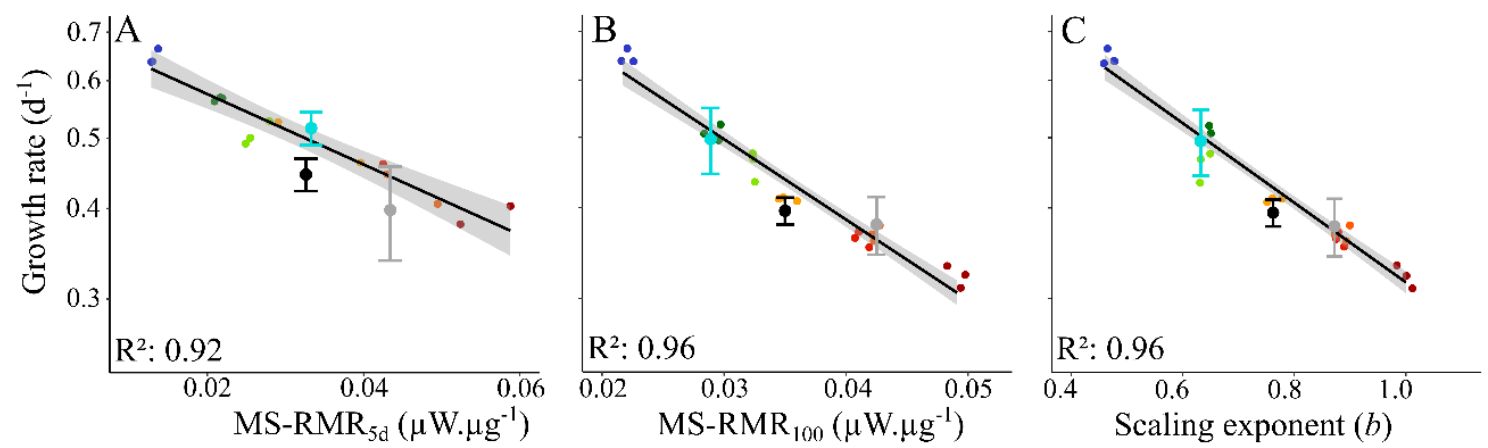

\begin{tabular}{|c|c|c|c|c|}
\hline \multicolumn{5}{|c|}{ Dietary treatments } \\
\hline . & $\begin{array}{l}\square \text { Cryptomonas sp. P+ } \\
\square \text { Chlamydomonas reinhardtii P+ } \\
\square \text { Chlamydomonas reinhardtii P- }\end{array}$ & $\begin{array}{l}\square \text { Synechococcus sp. }+12 \mu \mathrm{g} \text { cholesterol } \\
\square \text { Synechococcus sp. }+8 \mu \mathrm{g} \text { cholesterol } \\
\square \text { Synechococcus sp. }+4 \mu \mathrm{g} \text { cholesterol } \\
\square \text { Synechococcus sp. }+2 \mu \mathrm{g} \text { cholesterol }\end{array}$ & 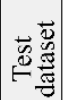 & $\begin{array}{l}\square \text { Cryptomonas sp. P- } \\
\square \text { Mixed diet (Syn } 80 \% / \mathrm{Chl} 20 \% \text { ) } \\
\square \text { Natural seston }\end{array}$ \\
\hline
\end{tabular}

619 Figure 2: Daphnids juvenile growth rate (from birth to maturity) versus the mass specific $620 R M R$ of 5 days old individuals (MS-RMR ${ }_{5 d}$ - panel A), versus MS-RMR of $100 \mu \mathrm{g}$ 621 individuals $\left(M S-R M R_{100}\right.$ - panel B) or versus the scaling exponent b (panel $\left.C\right)$. In each 622 panel, the black line is the linear model fitted from the training dataset with its 95\%CI 623 (shaded area). For the 7 treatments of the training dataset each point reflects the 624 measurements of one replicate jar. The 3 diets from the test dataset are reported with their 625 mean values and $95 \%$ CI. 

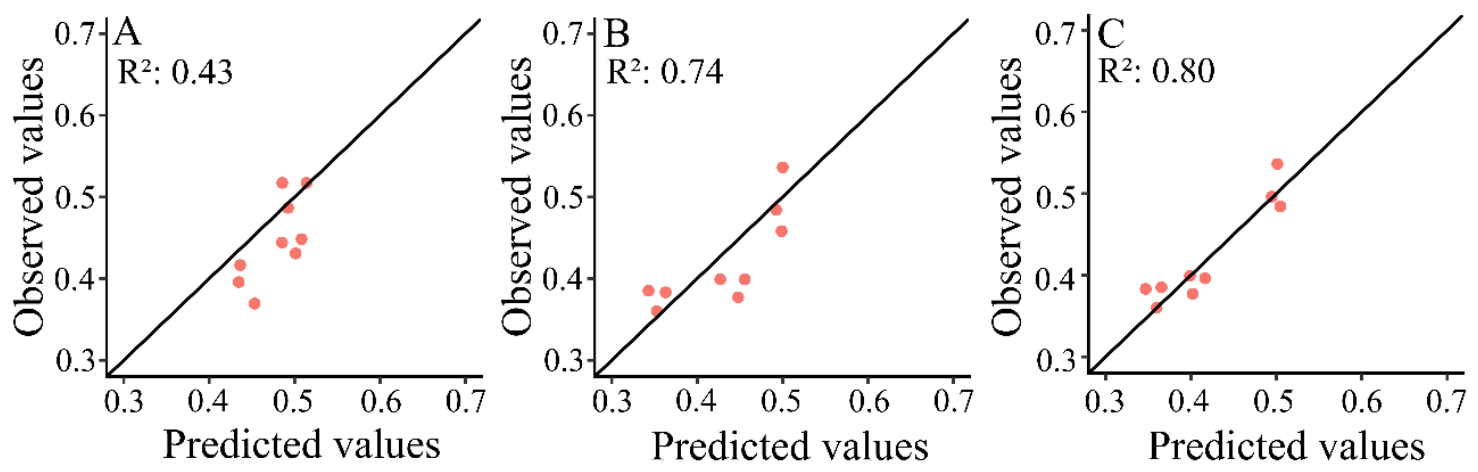

629 Figure 3: Observed versus predicted growth rates of the test dataset. Growth rate were 630 predicted using the linear models of figure 2 linking growth with MS-RMR 5 (panel A), $631 M S-R M R_{100}$ (panel $B$ ) or scaling exponent $b$ (panel $C$ ). The correlation between predicted 632 and observed values is given as $R^{2}$. Black line reflects the 1:1 line. 


\begin{tabular}{|c|c|c|c|c|c|c|c|}
\hline & \multirow{2}{*}{ Dietary Treatment } & \multicolumn{2}{|c|}{ HUFA } & \multicolumn{2}{|c|}{ PUFA } & \multirow{2}{*}{ Sterol } & \multirow{2}{*}{$\mathrm{C}: \mathrm{P}$ ratio } \\
\hline & & $20: 5 n-3$ & $22: 6 n-3$ & $18: 2 n-6$ & $18: 3 n-3$ & & \\
\hline \multirow{7}{*}{ 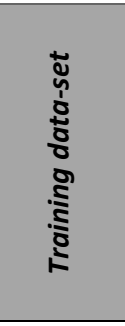 } & Cryptomonas sp. P+ & $8.8 \pm 1.2$ & $2.7 \pm 0.5$ & $7.9 \pm 0.1$ & $8.4 \pm 0.4$ & $10-12 *$ & $111.9 \pm 6.7$ \\
\hline & Chlamydomonas sp. $\mathrm{P+}$ & Und. & Und. & $14.5 \pm 0.3$ & $10.9 \pm 0.2$ & $3-5^{*}$ & $166.3 \pm 4.1$ \\
\hline & Chlamydomonas sp. P- & Und. & Und. & $27.4 \pm 5.7$ & $11.2 \pm 1.9$ & $3-5^{*}$ & $\begin{array}{c}1439.6 \pm \\
174.8\end{array}$ \\
\hline & Synechococcus $+12 \mu \mathrm{g}$ cholesterol & Und. & Und. & Und. & Und. & 12 & $195 \pm 6.9$ \\
\hline & Synechococcus $+8 \mu \mathrm{g}$ cholesterol & Und. & Und. & Und. & Und. & 8 & $195 \pm 6.9$ \\
\hline & Synechococcus $+4 \mu \mathrm{g}$ cholesterol & Und. & Und. & Und. & Und. & 4 & $195 \pm 6.9$ \\
\hline & Synechococcus $+2 \mu g$ cholesterol & Und. & Und. & Und. & Und. & 2 & $195 \pm 6.9$ \\
\hline \multirow{3}{*}{ 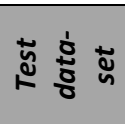 } & Cryptomonas sp. $P$ - & $7.6 \pm 0.7$ & $2.1 \pm 0.4$ & $8.3 \pm 0.4$ & $8 \pm 0.2$ & $10-12$ * & $453.5 \pm 59.4$ \\
\hline & Mixed diet & Und. & Und. & 2.9 & 2.2 & $0.6-1^{*}$ & 171.8 \\
\hline & Natural seston & Und. & Und. & $4.7 \pm 1.1$ & $3.2 \pm 0.8$ & N.A. & $76.4 \pm 3.8$ \\
\hline
\end{tabular}

637 Table 1. Dietary treatments used in the experiment and their main characteristics in term of 638 biochemical and stoichiometric composition. Fatty acid and sterol contents are given in $\mu$ g.mg $C^{-1}$. 639 Und. stands for undetected compounds and N.A. for not measured parameters. Values with 640 asterisks were taken from Martin-Creuzburg \& Merkel (2016).

641 